\title{
Analysis of light noise sources in a recycled Michelson interferometer with Fabry-Perot arms
}

\author{
J. B. Camp, H. Yamamoto, and S. E. Whitcomb \\ LIGO Project, California Institute of Technology, MS 18-34, Pasadena, California 91125 \\ D. E. McClelland \\ Department of Physics, The Faculties, The Australian National University, Science Road, Canberra, \\ ACT 0200, Australia
}

\begin{abstract}
Received March 29, 1999; revised manuscript received September 14, 1999; accepted September 15, 1999
We present a method by which the effect of laser field variations on the signal output of an interferometric gravitational wave detector is rigorously determined. Using the Laser Interferometer Gravitational Wave Observatory (LIGO) optical configuration of a power recycled Michelson interferometer with Fabry-Perot arm cavities as an example, we calculate the excess noise after the input filter cavity (mode cleaner) and the dependence of the detector strain sensitivity on laser frequency and amplitude noise, radio frequency oscillator noise, and scattered-light phase noise. We find that noise on the radio frequency sidebands generally limits the detector's sensitivity. (c) 2000 Optical Society of America [S0740-3232(00)01401-0]
\end{abstract}

OCIS codes: $120.2230,120.3180$.

\section{INTRODUCTION}

The search for astrophysical sources of gravitational radiation will employ long-baseline laser interferometers. These include the Laser Interferometer Gravitational Wave Observatory $^{1}$ (LIGO), the VIRGO project, $^{2}$ the TAMA300 project, ${ }^{3}$ and the GEO600 project. ${ }^{4}$ All of these will employ a variant of a Michelson interferometer illuminated with stabilized laser light. The light will be phase modulated at radio frequency (rf), producing modulation sidebands about the carrier frequency that provide a phase reference for sensing small variations of the interferometer arm lengths. ${ }^{5}$ Gravitational radiation will produce a differential length change of the arms of the Michelson interferometer, causing a signal at the output port.

In Fig. 1 we show the configuration of the LIGO detector. The light from a stabilized laser source is phase modulated before it enters a triangular filter cavity (referred to as a mode cleaner), which is tuned to transmit only the TEM $\mathrm{M}_{00}$ spatial mode of the beam. The light then enters the interferometer, which comprises an asymmetric Michelson interferometer with Fabry-Perot arm cavities. The use of arm cavities serves to resonantly enhance the phase shift of light caused by a change in arm length. An asymmetry in the Michelson lengths transmits the rf sidebands to the output photodetector, where they beat against carrier light that is phase shifted from a differential arm length change. Demodulation of the photocurrent at the modulation frequency gives an output signal. An additional mirror placed between the laser and the beam splitter increases the total light power available to the arms by forming a recycling cavity to- gether with the beam splitter and arm cavity input mirrors. ${ }^{6}$

The presence of noise at the interferometer output must be held below the desired strain sensitivity. The primary noise sources that define the interferometer's sensitivity are seismic noise at frequencies below $100 \mathrm{~Hz}$, thermal noise roughly from 100 to $300 \mathrm{~Hz}$, and photon counting noise at frequencies greater than $300 \mathrm{~Hz}$. Light-noise sources are those that, if properly suppressed, will not limit the strain sensitivity. In particular, variations in the phase and amplitude of the laser electric field used to probe the cavity lengths must be carefully controlled, because small interferometer imperfections such as differential arm cavity losses and deviation from fringe center of the cavity lengths can cause spurious phase shifts and couple to the light noise to produce an output at the gravitational wave (gw) signal output.

In this paper we calculate the induced noise from the following sources of noise on the laser light: frequency, amplitude, rf oscillator, and backscattering from the vacuum enclosure. The approach is to write the noise as frequency components of the light, phase modulate the light, and propagate the resultant frequency spectrum through the mode cleaner and to the interferometer signal port, carefully evaluating the resultant phase shifts encountered. We then evaluate the light intensity at the photodetector and demodulate the resultant output. This approach is used to investigate noise couplings in the first-generation LIGO interferometer but may be applied to other interferometer configurations. In Section 2 we show the effect of the noise on the frequency spectrum of the light, including both the carrier and the rf sideband 


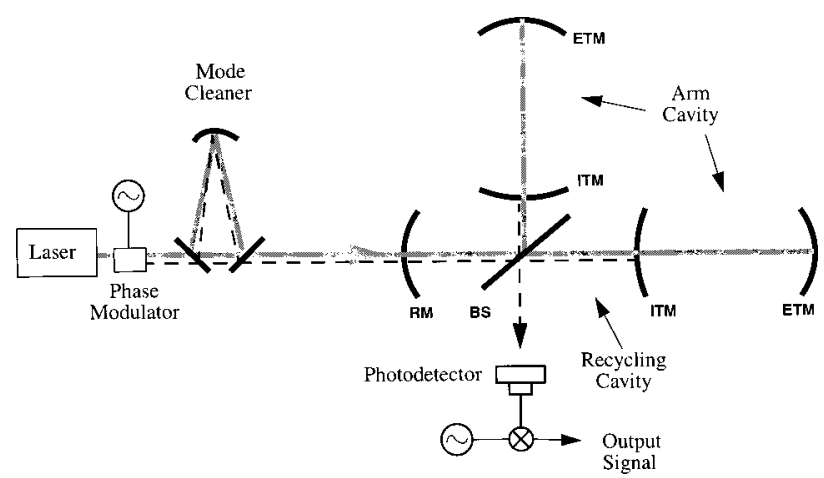

Fig. 1. Schematic of the detector showing laser, phase modulator, mode-cleaner filter cavity, and interferometer, including the recycling cavity (formed by recycling mirror RM, beam splitter BS, and input test mass ITM) and Fabry-Perot arm cavities (formed by ITM and end test mass ETM). Carrier light (solid lines) and sideband light (dashed lines) are shown offset for clarity. A differential arm length change will combine carrier and sidebands at the gw port, where they are detected and demodulated to yield the output signal. Lengths are not drawn to scale.

frequencies. In Section 3 we derive the transfer function the interferometer signal output, and in Section 4 we compare the resultant noise after the mode cleaner with shot noise and at the interferometer gw output with the interferometer's sensitivity. We find that noise on the carrier and on rf sidebands undergo different filtering in the interferometer and that the rf noise generally dominates the gw output noise.

\section{NOISE SPECTRUM OF LIGHT}

The light noise that we consider can be written as audio range $(0-10-k H z)$ frequency components about the carrier frequency $\omega_{0}$, which produce additional audio sidebands when the light is phase modulated at the $\mathrm{rf} \Omega$. We first look at frequency noise. The electric field of the (noiseless) light is

$$
E=E_{0} \exp \left(i \omega_{0} t\right),
$$

where $\mathrm{E}_{0}$ and $\omega_{0}$ are the carrier amplitude and frequency, respectively. Writing frequency noise as $\nu=\nu_{0}$ $+\delta \nu \cos \omega t$, where $\delta \nu$ and $\omega$ are the amplitude and the Fourier frequency of the frequency variation, respectively, we find the total laser el ectric field $E_{L}$ phase variation:

$$
\phi_{\mathrm{L}}(\mathrm{t})=2 \pi v_{0} \mathrm{t}+\frac{2 \pi \delta v}{\omega} \sin \omega \mathrm{t} .
$$

Thus we can write

$$
\begin{aligned}
\mathrm{E}_{\mathrm{L}}= & \mathrm{E}_{0}\left(\exp \left(\mathrm{i} \omega_{0} \mathrm{t}\right)+\frac{\pi \delta v}{\omega}\left\{\exp \left[\mathrm{i}\left(\omega_{0}+\omega\right) \mathrm{t}\right]\right.\right. \\
& \left.\left.-\exp \left[\mathrm{i}\left(\omega_{0}-\omega\right) \mathrm{t}\right]\right\}\right) .
\end{aligned}
$$

Now the laser electric field $E_{L}$ is phase modulated at $\mathrm{rf} \Omega$ and depth $\Gamma$ to produce rf sidebands, yielding the final laser incident electric field $E_{i}$ :

$$
\begin{aligned}
\mathrm{E}_{\mathrm{i}} & =\mathrm{E}_{\mathrm{L}} \exp \left(\mathrm{i}_{\text {osd }}\right), \quad \mathrm{A}_{\text {osc }}=\Gamma \cos \Omega \mathrm{t} \\
& =\mathrm{E}_{\mathrm{L}}\left\{1+\frac{\mathrm{i} \Gamma}{2}[\exp (\mathrm{i} \Omega \mathrm{t})+\exp (-\mathrm{i} \Omega \mathrm{t})]\right\}, \quad \Gamma \ll 1 ;
\end{aligned}
$$

then

$$
\begin{aligned}
\mathrm{E}_{\mathrm{i}}= & \mathrm{E}_{0}\left(\exp \left(\mathrm{i} \omega_{0} \mathrm{t}\right)+\frac{\pi \delta v}{\omega}\left\{\exp \left[\mathrm{i}\left(\omega_{0}+\omega\right) \mathrm{t}\right]\right.\right. \\
& \left.-\exp \left[\mathrm{i}\left(\omega_{0}-\omega\right) \mathrm{t}\right]\right\} \\
& +\frac{\mathrm{i} \Gamma}{2}\left\{\exp \left[\mathrm{i}\left(\omega_{0}+\Omega\right) \mathrm{t}\right]+\exp \left[\mathrm{i}\left(\omega_{0}-\Omega\right) \mathrm{t}\right]\right\} \\
& +\frac{\mathrm{i} \pi \delta v \Gamma}{2 \omega}\left\{\exp \left[\mathrm{i}\left(\omega_{0}+\omega+\Omega\right) \mathrm{t}\right]\right. \\
& -\exp \left[\mathrm{i}\left(\omega_{0}-\omega+\Omega\right) \mathrm{t}\right]+\exp \left[\mathrm{i}\left(\omega_{0}+\omega-\Omega\right) \mathrm{t}\right] \\
& \left.\left.-\exp \left[\mathrm{i}\left(\omega_{0}-\omega-\Omega\right) \mathrm{t}\right]\right\}\right) .
\end{aligned}
$$

Equation (5) contains nine frequency components: the carrier and $2 \mathrm{rf}$ sidebands and their associated noise audio sidebands. Figure 2 shows the frequency components of the light, including the noise audio sidebands; here $\mathrm{E}_{\mathrm{ab}}$ refers to the electric field component of rf index $a$ and audio index $b$.

In a similar manner, we can construct the frequency spectrum for the following light-noise sources with the following substitutions for $E$ or $A_{\text {osc }}$ : laser amplitude noise, $\mathrm{E}=\mathrm{E}_{0}+\delta \mathrm{E} \cos \omega \mathrm{t}$; $r$ oscillator phase noise, $\mathrm{A}_{\text {osc }}$ $=\Gamma \cos \left(\Omega t+a_{0} \cos \omega t\right)$; rf oscillator amplitude noise, $\mathrm{A}_{\text {osc }}=\Gamma[1+(\delta \mathrm{A} / \mathrm{A}) \cos \omega \mathrm{t}] \cos \Omega \mathrm{t}$.

We also consider the variation in the light from phase noise produced by carrier and sideband modulation in stray light scattering into the recycling cavity or arm cavity. Here the noise is placed on the light after the rf modulation, as the light path is modulated by the motion of the scattering surface. Phase modulation of both carrier and rf sideband takes place as light is backscattered into the recycling cavity from the output port photodiode surface, yielding the electric field from phase noise $E_{p n}$ :

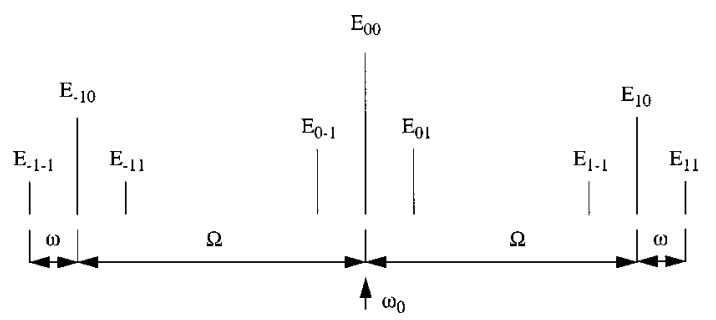

Fig. 2. Frequency spectrum of light, showing audio noise sidebands about both carrier and $r f$ sideband frequencies. $E_{a b}$, electric field component of $r f$ index $a$ and audio index $b$. 
Table 1. Frequency Spectrum of Noise Sources

\begin{tabular}{|c|c|c|c|c|c|c|c|c|c|c|}
\hline $\begin{array}{l}\text { Noise } \\
\text { Source }\end{array}$ & $E_{-1-1}$ & $E_{-10}$ & $E_{-11}$ & $\mathrm{E}_{0-1}$ & $E_{00}$ & $E_{01}$ & $E_{1-1}$ & $\mathrm{E}_{10}$ & $E_{11}$ & $\begin{array}{l}\text { Source } \\
\text { Factor }\end{array}$ \\
\hline $\begin{array}{l}\text { Laser } \\
\text { frequency }\end{array}$ & $-\frac{\mathrm{i} \Gamma \pi \delta \mathrm{v}}{2 \omega}$ & $\frac{\mathrm{i} \Gamma}{2}$ & $\frac{\mathrm{i} \Gamma \pi \delta \mathrm{v}}{2 \omega}$ & $\frac{-\pi \delta \mathrm{v}}{\omega}$ & 1 & $\frac{\pi \delta \mathrm{v}}{\omega}$ & $\frac{-\mathrm{i} \Gamma \pi \delta \mathrm{v}}{2 \omega}$ & $\frac{\mathrm{i} \Gamma}{2}$ & $\frac{\mathrm{i} \Gamma \pi \delta \mathrm{v}}{2 \omega}$ & $E_{0}$ \\
\hline $\begin{array}{l}\text { Laser } \\
\text { amplitude }\end{array}$ & $\frac{\mathrm{i} \Gamma \delta \mathrm{E}}{4 \mathrm{E}_{0}}$ & $\frac{\mathrm{i} \Gamma}{2}$ & $\frac{\mathrm{i} \Gamma \delta \mathrm{E}}{4 \mathrm{E}_{0}}$ & $\frac{\delta \mathrm{E}}{2 \mathrm{E}_{0}}$ & 1 & $\frac{\delta \mathrm{E}}{2 \mathrm{E}_{0}}$ & $\frac{\mathrm{i} \Gamma \delta \mathrm{E}}{4 \mathrm{E}_{0}}$ & $\frac{\mathrm{i} \Gamma}{2}$ & $\frac{\mathrm{i} \Gamma \delta \mathrm{E}}{4 \mathrm{E}_{0}}$ & $\mathrm{E}_{0}$ \\
\hline $\begin{array}{l}\text { Oscillator } \\
\text { phase }\end{array}$ & $\frac{\Gamma \mathrm{a}_{0}}{4}$ & $\frac{i \Gamma}{2}$ & $\frac{\Gamma \mathrm{a}_{0}}{4}$ & - & 1 & - & $\frac{-\Gamma \mathrm{a}_{0}}{4}$ & $\frac{\mathrm{i} \Gamma}{2}$ & $\frac{-\Gamma \mathrm{a}_{0}}{4}$ & $\mathrm{E}_{0}$ \\
\hline $\begin{array}{l}\text { Oscillator } \\
\text { amplitude }\end{array}$ & $\frac{\mathrm{i} \Gamma \delta \mathrm{A}}{4 \mathrm{~A}}$ & $\frac{\mathrm{i} \Gamma}{2}$ & $\frac{\mathrm{i} \Gamma \delta \mathrm{A}}{4 \mathrm{~A}}$ & - & 1 & - & $\frac{\mathrm{i} \Gamma \delta \mathrm{A}}{4 \mathrm{~A}}$ & $\frac{\mathrm{i} \Gamma}{2}$ & $\frac{\mathrm{i} \Gamma \delta \mathrm{A}}{4 \mathrm{~A}}$ & $E_{0}$ \\
\hline $\begin{array}{l}\text { Dark port } \\
\text { scattered } \\
\text { light }\end{array}$ & $\frac{-\Gamma k_{0} x_{s}}{2}$ & $\frac{\mathrm{i} \Gamma}{2}$ & $\frac{-\Gamma k_{0} x_{S}}{2}$ & $i k_{0} x_{s}$ & 1 & $i k_{0} x_{s}$ & $\frac{-\Gamma k_{0} x_{s}}{2}$ & $\frac{\mathrm{i} \Gamma}{2}$ & $\frac{-\Gamma k_{0} x_{s}}{2}$ & $E_{\text {scat }}$ \\
\hline $\begin{array}{l}\text { Arm } \\
\text { scattered } \\
\text { light }\end{array}$ & - & - & - & $i k_{0} x_{s}$ & 1 & $i k_{0} x_{s}$ & - & - & - & $E_{\text {scat }}$ \\
\hline
\end{tabular}

$$
\begin{aligned}
E_{p n}= & E_{s c a t} \exp \left(i \omega_{0} t\right) \\
& \times\left\{1+\frac{i \Gamma}{2}[\exp (i \Omega t)+\exp (-i \Omega t)]\right\} \\
& \times\left\{\exp \left[i 2 k x_{s} \cos (\omega t)\right]\right\} \\
= & E_{s c a t} \exp \left(i \omega_{0} t\right) \\
& \times\left\{1+\frac{i \Gamma}{2}[\exp (i \Omega t)+\exp (-i \Omega t)]\right\} \\
& \times\left\{1+i k x_{s}[\exp (i \omega t)+\exp (-i \omega t)]\right\}
\end{aligned}
$$

for $k x_{s} \ll 1$, where $E_{\text {scat }}$ is the amplitude of the scattered light, $x_{s} \cos (\omega t)$ is the seismic motion of the photodiode, and $k$ is the carrier wave number. In arm cavity scattering the rf sidebands are not present, so the rf components in expression (6) are removed.

The frequency spectrum that corresponds to each of these noise sources is listed in Table 1 . The rightmost column of the table gives the overall source factor by which all amplitudes in the row that correspond to a noise source are multiplied.

\section{TRANSFER FUNCTION OF THE LIGHT- FREQUENCY SPECTRUM THROUGH THE MODE-CLEANER CAVITY AND INTERFEROMETER}

\section{A. Mode Cleaner}

We propagate the light frequency spectrum for laser or oscillator noise through the mode cleaner in the following way: Taking the reflectivity of the curved mirror to be unity, we write the equilibrium cavity field equations ${ }^{7}$ (see Fig. 3) as

$$
\begin{aligned}
E_{t} & =t_{m} E_{i}-r_{m} E_{r}, \\
E_{r} & =E_{t} \exp (-i \Phi) r_{m}, \\
E_{m} & =E_{t} \exp (-i \Phi / 2) t_{m},
\end{aligned}
$$

where $\Phi$ is the round-trip phase change impressed on the light by its traversing the cavity; $\mathrm{t}_{\mathrm{m}}$ and $\mathrm{r}_{\mathrm{m}}$ are the am-

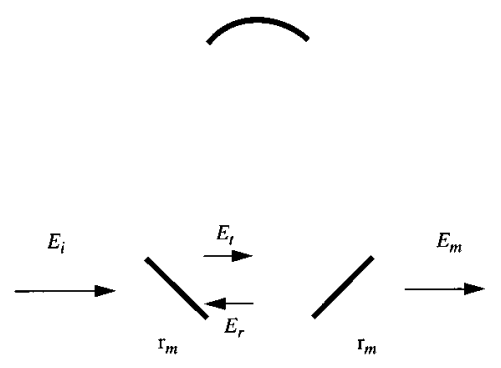

Fig. 3. Diagram of mode cleaner, showing input and output mirror reflectivities and equilibrium fields. We take the curved mirror reflectivity to be unity.

plitude transmission and reflection of the mode cleaner input and output mirrors, respectively; and $E_{i}$ and $E_{m}$ are the input and output fields, respectively. The equations may be combined to give the steady-state output field $\mathrm{E}_{\mathrm{m}}$ :

$$
E_{m}=\frac{t_{m}^{2} E_{i} \exp (-i \Phi / 2)}{1+r_{m}^{2} \exp (-i \Phi)}
$$

The round-trip phase for the frequency components is calculated as follows: The mode cleaner's length must be held in resonance with both the carrier and the rf sideband frequencies to allow transmission of the light to the interferometer. The resonance conditions are

$$
\begin{aligned}
2 \mathrm{~kL} & =(2 \mathrm{~m}+1) \pi, \\
\frac{2 \mathrm{~L}_{\mathrm{m}} \Omega}{\mathrm{c}} & =(2 \mathrm{n}) \pi
\end{aligned}
$$

where $m$ and $n$ are integers, $L_{m}$ is the mode cleaner's resonant length, and $\Omega$ is the rf. Including the modecleaner imperfections of a root-mean-square (rms) length offset from resonance $L_{m}^{\prime}=L_{m}+d x_{m}$ and a rms sideband detuning from resonance $d \Omega$, we have 


$$
\begin{aligned}
\Phi^{C} & =2 k L_{m}^{\prime}=\frac{2}{c}\left(\omega_{0}+\omega\right)\left(L_{m}+d x_{m}\right) \\
& =\pi+\frac{2}{c}\left(\omega L_{m}+\omega_{0} d x_{m}\right), \\
\Phi^{S} & =2\left(k+\frac{\Omega+d \Omega}{C}\right) L_{m}^{\prime} \\
& =\pi+\frac{2}{c}\left(\omega L_{m}+\omega_{0} d x_{m}+d \Omega L_{m}\right),
\end{aligned}
$$

where $\omega$ is the audio frequency of the noise source under consideration. The phase shifts are calculated for each of the nine frequency components of a noise source.

We insert these expressions into Eq. (10). We take the near-resonance approximation:

$$
\exp (\mathrm{i} \Phi) \sim 1+\mathrm{i} \Phi
$$

We obtain

$$
\begin{aligned}
& E_{m}{ }^{c}=i E_{i} c\left\{\frac{1+i\left(\frac{\omega L_{m}}{c}+k d x_{m}\right)}{\left[1+2 i G_{m}\left(\frac{\omega L_{m}}{c}+k d x_{m}\right)\right]}\right\}^{\prime} \\
& E_{m}{ }^{s}=i E_{i} s\left\{\frac{1+i\left(\frac{\omega L_{m}}{c}+k d x_{m}+\frac{d \Omega L_{m}}{c}\right)}{\left[1+2 i G_{m}\left(\frac{\omega L_{m}}{c}+k d x_{m}+\frac{d \Omega L_{m}}{c}\right)\right]}\right\}
\end{aligned}
$$

for $E_{m}^{c}\left(E_{m}{ }^{s}\right)$, the amplitudes of the carrier (sideband) frequency components after the mode cleaner (where we now refer in superscript to carrier or rf sideband, each understood to have two associated audio sidebands). Here $\mathrm{G}_{\mathrm{m}}=1 /\left(1-\mathrm{r}_{\mathrm{m}}^{2}\right)$ and $\mathrm{E}_{\mathrm{i}}^{\mathrm{c}}$ and $\mathrm{E}_{\mathrm{i}}^{\mathrm{s}}$ are the amplitudes of the frequency components (product of the the amplitude and the source factor) listed in Table 1, rows 1-4.

With the approximation that $\omega \ll \omega_{\mathrm{m}}$ for most of the audio frequency band, where $\omega_{m}=c /\left(2 G_{m} L_{m}\right)$ (the mode-cleaner cavity pole frequency), E qs. (16) and (17) reduce to

$$
\begin{aligned}
& E_{m}{ }^{c}=i E_{i}{ }^{c}\left[1-2 i G_{m}\left(\frac{\omega L_{m}}{c}+k d x_{m}\right)\right], \\
& E_{m}{ }^{s}=i E_{i} s\left[1-2 i G_{m}\left(\frac{\omega L_{m}}{c}+k d x_{m}+\frac{d \Omega L_{m}}{c}\right)\right] .
\end{aligned}
$$

We see that the effect of the mode-cleaner imperfections of $r$ s fringe offset $d x_{m}$ or sideband detuning $d \Omega$ is to impose phase shifts on the frequency components of the noise sources. A relative phase shift of carrier and sideband can appear as excess noise after demodulation (evaluated below).

\section{B. Interferometer}

We continue with the propagation of the fields of E qs. (18) and (19) to the interferometer output port. In Fig. 4 we show the interferometer mirrors and input and output fields and the recycling cavity lengths (defined as the distances from the recycling mirror to the two-arm cavity input mirrors.) We take the reflectivities of the interferometer end mirrors to be unity.

After exiting the mode cleaner, the fields are incident on the power recycling mirror. The beam splitter is held at a position such that the carrier power at the output port (also called the dark port) is minimized. The average recycling length is chosen to allow the sidebands to resonate in the recycling cavity; the lengths are chosen to be different by an amount $\delta$ (referred to as the interferometer asymmetry) that allows transmission of the sidebands to the dark port. With the approximation that the recycling mirror and the beam splitter are close, we have ${ }^{8}$

$$
E_{d}=\frac{E_{m}}{2} t_{r} \frac{\left[r_{2} \exp \left(-i \phi_{2}\right)-r_{1} \exp \left(-i \phi_{1}\right)\right]}{1+\frac{r_{r}}{2}\left[r_{1} \exp \left(-i \phi_{1}\right)+r_{2} \exp \left(-i \phi_{2}\right)\right]},
$$

where $\phi_{1}$ and $\phi_{2}$ are the phase shifts traversed over the round-trip recycling cavity lengths, $E_{d}$ is the field at the dark port, $t_{r}$ is the recyding mirror transmission, and $r_{1}$ and $r_{2}$ are the complex arm cavity reflectivities. We now evaluate this expression for the carrier and sideband fields, using the expansion of relation (15).

\section{Carrier}

The carrier light undergoes a $\pi$ phase shift on reflection from the arm cavities (Appendix A). Thus the resonance condition for the carrier in the recycling cavity is $\phi_{1}{ }^{\mathrm{C}}$ $=\phi_{2}{ }^{\mathrm{C}}=0$. Then

$$
E_{d}{ }^{c}=\frac{E_{m}{ }^{c}}{2} \frac{t_{r} \Delta r}{1+r_{r} r^{c}},
$$

where $\Delta r=r_{2}{ }^{c}-r_{1}{ }^{c}$ is the difference in carrier reflectivity for the arms. The circulating fields for the arm cavity give the following expression for the carrier arm reflectivity (see Appendix A):

$$
r^{c}=\frac{r 0+i r_{f} \frac{\omega}{\omega_{c}}+i G_{a} k d x_{a}}{1+\frac{i \omega}{\omega_{c}}},
$$

where $\mathrm{r} 0$ is the arm cavity carrier reflectivity at exact resonance, $G_{a}$ is the arm cavity optical gain, $d_{x}$ is the

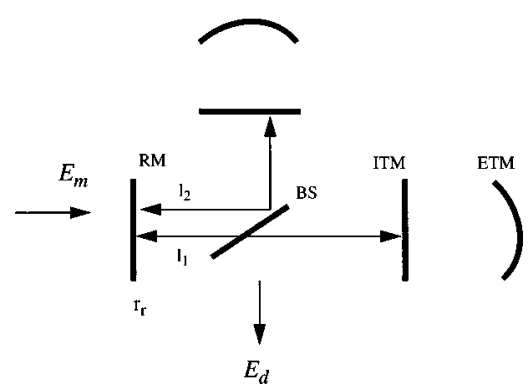

Fig. 4. Interferometer mirrors and input and output fields. $I_{1}$ and $\mathrm{I}_{2}$ are the recycling cavity lengths, $\mathrm{r}_{\mathrm{r}}$ is the recycling mirror reflectivity, and $E_{d}$ is the dark port field. ITM, input test mass; ETM, end test mass. 
arm cavity rms deviation from resonance, and $\omega_{c}$ is the arm cavity pole frequency. Insertion of Eq. (22) into Eq. (21) yields

$$
\begin{aligned}
E_{d}{ }^{c}= & \frac{E_{m}{ }^{c}}{2} t_{r} G_{r}{ }^{c} \\
& \times\left[\frac{A_{c m}\left(1+i r_{f} \frac{\omega}{\omega_{c}}\right)+i G_{a} k d x_{a-}+i k d x_{r}-}{\left(1+\frac{i \omega}{\omega_{c c}}\right)}\right],
\end{aligned}
$$

where $A_{c m}=r 0_{1}-r_{2}$, the arm cavity carrier reflectivity match at exact resonance that provides the interferometer's common mode attenuation of frequency noise, $\mathrm{dx}_{\mathrm{a}-}$ and $\mathrm{dx}_{\mathrm{r}}$ - are the arm and recycling cavity rms differential length deviations from resonance, and $G_{r}{ }^{c}$ $=1 /\left(1+r_{r} r 0\right)$ is the buildup of the carrier amplitude in the recycling cavity. The pole frequency of Eq. (23), $\omega_{\mathrm{cc}}$ $=\left(\omega_{c} / 2\right)\left(1+r_{r} r 0\right)$, arises from the coupling of the recycling and arm cavities, which gives a storage time for the light in the interferometer of order $\mathrm{G}_{r}{ }^{\mathrm{c}}$ times the arm cavity lifetime. We see that audio noise sidebands on the carrier are filtered by the coupled recycling-arm cavity.

\section{Radio Frequency Sidebands}

The sidebands, which are nonresonant in the arms, have an arm cavity reflectivity of unity. With the average recycling cavity length chosen such that $2 L_{\text {avg }}=(2 n+1)$ $\times(\pi \mathrm{c} / \Omega)$, the resonance condition for the sidebands, we find that

$$
\mathrm{E}_{\mathrm{d}} \mathrm{s}=-\mathrm{iE}_{\mathrm{m}} \mathrm{s}_{\mathrm{r}} \mathrm{G}_{\mathrm{r}} \mathrm{s}\left(\frac{\Omega \delta}{\mathrm{c}}+\frac{\omega \delta}{\mathrm{c}}+\mathrm{kdx}_{\mathrm{r}-}\right),
$$

where $\delta$ is the difference in length from the recycling mirror to either arm cavity and $\mathrm{G}_{\mathrm{r}}{ }^{\mathrm{s}}=1 /\left[1-\mathrm{r}_{\mathrm{r}} \cos (\Omega \delta / \mathrm{c})\right]$ is the sideband amplitude buildup in the recycling cavity and where we have omitted the recycling-cavity pole frequency $\omega_{\mathrm{rc}}=\left(1-\mathrm{r}_{\mathrm{r}}\right)\left(\mathrm{c} / 2 \mathrm{~L}_{\mathrm{avg}}\right)$, which is large compared with the audio frequencies that we are considering. Audio noise sidebands on the rf sidebands thus propagate to the dark port with no filtering.

\section{Scattered Light}

Scattered light enters the interferometer through a different path and with a source term different from that of Subsection 3.B. We consider light scattered from the dark-port photodetector back onto the beam splitter and from the interferometer vacuum enclosure back into the arm. Here we use the frequency spectrum of Eq. (6), which corresponds to rows 5 and 6 of Table 1 .

\section{Dark Port}

Scattered light injected back into the interferometer at this location couples to the recyding cavity in the following way: The recycling cavity is essentially transparent to the sidebands, so the scattered sidebands are transmitted through the recycling mirror. However, the carrier is split by the beam splitter, reflected from the arms, and recombined constructively with almost no buildup or common-mode attenuation. For the carrier we set $G_{r}{ }^{c}$ $=1$ and $A_{c m}=1$ and apply the filtering of the single arm cavity pole. We obtain

$$
E_{d}^{c}=E_{p n} c\left[\frac{1+i r_{f} \frac{\omega}{\omega_{c}}+i G_{a} k d x_{a-}+i k d x_{r-}}{\left(1+\frac{i \omega}{\omega_{c}}\right)}\right] \text {. }
$$

The remaining rf sideband field at the dark port, absent noise audio sidebands, is

$$
\mathrm{E}_{\mathrm{d}}^{\mathrm{s}}=-\mathrm{E}_{\mathrm{m}} \mathrm{s}_{\mathrm{r}} \mathrm{G}_{\mathrm{r}} \mathrm{si}\left(\frac{\Omega \delta}{\mathrm{c}}+\mathrm{kdx}_{\mathrm{r}-}\right),
$$

where the source factor for the rf sidebands in this term is $\mathrm{E}_{0}$.

\section{Arm Cavity}

Here the scattered carrier light enters directly into the arm cavity. We use Eq. (B2) of Appendix B and replace source term $E_{s}$ with $E_{p n}$. We obtain

$$
E_{d}{ }^{c}=\frac{E_{p n}{ }^{c}}{2} \frac{t_{f} G_{a}}{\left(1+\frac{i}{\omega_{c}}\right)} .
$$

The sideband field is given by Eq. (26).

\section{NOISE COUPLINGS}

In this section we evaluate the effect of the noise inputs on the signals detected after the mode cleaner and at the interferometer dark port.

\section{A. Noise Couplings after the Mode Cleaner}

We look at the excess noise relative to shot noise on the total light following the mode cleaner generated by each noise source by evaluating the electric field components on the photodetector according to Table 1 and Eqs. (18) and (19) and then demodulating the photodetector current according to Appendix C. (We used a Mathematica code to symbolically evaluate and reduce the cumbersome expressions.)

For example, the full-power photocurrent detected after the mode cleaner as a result of laser frequency noise is

$$
\mathrm{i}_{\mathrm{p}}=8 \epsilon \frac{\mathrm{e}}{\mathrm{h} v_{0}} \Gamma \mathrm{PG}_{\mathrm{m}}{ }^{2} \pi\left(\frac{\mathrm{L}_{\mathrm{m}}}{\mathrm{c}}\right)^{2}(\delta v \mathrm{~d} \Omega),
$$

where $\epsilon$ is the photodetector's quantum efficiency, $h$ is Planck's constant, e is the electric charge, and P is the laser power. Equation (28) shows that laser frequency noise couples to a sideband detuned from the modecleaner resonance. The signal from shot noise for the light exiting the mode cleaner is

$$
\mathrm{I}_{\mathrm{s}}=\mathrm{e}\left(\frac{2 \mathrm{P} \epsilon}{\mathrm{h} v_{0}}\right)^{1 / 2}
$$

Table 2 lists the mode-cleaner imperfections to which the noise sources couple and the noise levels of these sources that produce excess output noise equal to shot noise for 
Table 2. Noise Couplings after Mode Cleaner at $100 \mathrm{~Hz}$

\begin{tabular}{lccc}
\hline Noise Source & Coupling & $\begin{array}{c}\text { Demodulation } \\
\text { Phase }\end{array}$ & $\begin{array}{c}\text { Noise Level with } \\
\text { Excess } \\
\text { Noise Equal } \\
\text { to Shot Noise }\end{array}$ \\
\hline Laser frequency & $\mathrm{d} \Omega$ & $\mathrm{Q}$ & $8 \times 10^{-5} \mathrm{~Hz} / \sqrt{\mathrm{Hz}}$ \\
Laser amplitude & $\mathrm{d} \Omega \times \mathrm{dx}$ & $\mathrm{Q}$ & $2 \times 10^{-5} / \sqrt{\mathrm{Hz}}$ \\
Oscillator phase & $\omega \times \mathrm{dx}$ & $\mathrm{Q}$ & $3 \times 10^{-5} \mathrm{rad} / \sqrt{\mathrm{Hz}}$ \\
Oscillator & $\mathrm{d} \Omega \times \mathrm{dx}$ & $\mathrm{Q}$ & $3 \times 10^{-5} / \sqrt{\mathrm{Hz}}$ \\
amplitude & $\omega \times \mathrm{dx} \mathrm{m}_{\mathrm{m}}$ & $\mathrm{I}$ & $3 \times 10^{-5} / \sqrt{\mathrm{Hz}}$ \\
\hline
\end{tabular}

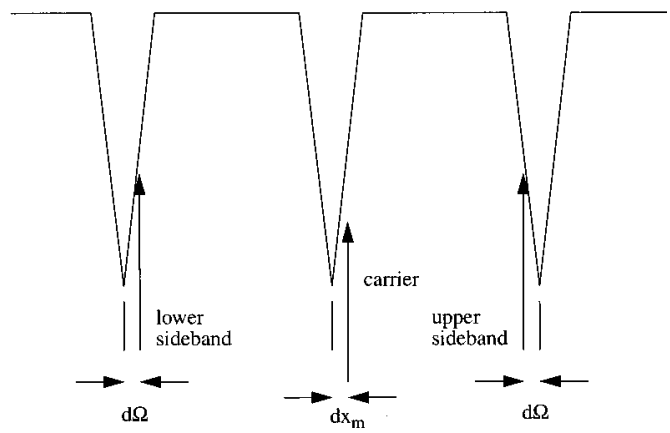

Fig. 5. Offset of frequency components from mode-cleaner resonances. The curve plots reflected intensity versus frequency of the incident light.

Table 3. Noise Couplings at GW Output at $100 \mathrm{~Hz}$

\begin{tabular}{lcc}
\hline Noise Source & Coupling & $\begin{array}{c}\text { Noise Level } \\
\text { with } \mathrm{GW} \\
\text { Output Noise } \\
\text { of } 10^{-20} \mathrm{~m} / \sqrt{\mathrm{Hz}}\end{array}$ \\
\hline Laser frequency & $\mathrm{A}_{\mathrm{cm}}$ & $2 \times 10^{-7} \mathrm{~Hz} / \sqrt{\mathrm{Hz}}$ \\
Laser amplitude & $\mathrm{dx}_{\mathrm{a}-}$ & $1 \times 10^{-7} / \sqrt{\mathrm{Hz}}$ \\
$\mathrm{dx}_{\mathrm{r}-}$ & $1 \times 10^{-7} / \sqrt{\mathrm{Hz}}$ \\
Oscillator phase & $\omega \times \mathrm{dx}_{\mathrm{a}-}$ & $2 \times 10^{-2} \mathrm{rad} / \sqrt{\mathrm{Hz}}$ \\
modulation & $\omega \times \mathrm{A}_{\mathrm{cm}} \times \mathrm{dx} \mathrm{r}_{\mathrm{r}}$ & $2 \times 10^{-4} \mathrm{rad} / \sqrt{\mathrm{Hz}}$ \\
Oscillator phase & $\mathrm{None}$ & - \\
Oscmodulation & $\mathrm{dx}_{\mathrm{a}-}$ & $1 \times 10^{-7} / \sqrt{\mathrm{Hz}}$ \\
Dark port scatter & $\mathrm{dx}_{\mathrm{r}-}$ & $1 \times 10^{-7} / \sqrt{\mathrm{Hz}}$ \\
Arm cavity scatter & $\mathrm{x}_{\mathrm{s}}$ & $1 \times 10^{-12} \mathrm{~W}$ \\
\hline & $\mathrm{x}_{\mathrm{s}}$ & $1 \times 10^{-14} \mathrm{~W}$ \\
\hline
\end{tabular}

$6 \mathrm{~W}$ of light. We use the values of Table 4 in Appendix D for the mode-cleaner parameters and imperfections.

The approach outlined in this paper may be complemented in some cases by a more heuristic description. For example, the laser and oscillator frequency noise couplings may be suggested by Fig. 5. The curve shows the mode-deaner reflectivity plotted against frequency, with the carrier and upper and lower rf sidebands shown relative to their respective resonance peaks. The rf sidebands are detuned from the resonances by $\mathrm{d} \Omega$, and the carrier is offset by an amount $\mathrm{dx} \mathrm{x}_{\mathrm{m}}$. Laser frequency noise will result in a variation of all three frequencies about these positions, whereas oscillator noise will cause the sideband frequencies to vary. It is suggestive from the symmetry of Fig. 5 that laser frequency noise will couple to $d \Omega$ whereas oscillator noise will couple to $d x_{m}$. Experimental observations have been made of the couplings of laser and oscillator frequency noise ${ }^{9}$ listed in Table 3.

\section{B. Noise Couplings at Gravitational Wave Output}

We demodulate in quadrature phase the photodetector current produced by the electric fields exiting the dark port according to Eqs. (23) and (24) for laser and oscillator noise or Eqs. (25)-(27) for scattered light. Each noise source couples to a number of interferometer imperfections to produce strain noise. It is interesting that the laser frequency noise and amplitude noise are dominated by noise on the rf sidebands, which are not filtered by the coupled cavity.

The photocurrent detected at the dark port that is due to laser frequency noise is

$$
\mathrm{I}=\epsilon \frac{\mathrm{e}}{\mathrm{h} v_{0}} \Gamma \mathrm{Pt}_{\mathrm{r}}{ }^{2} \mathrm{G}_{\mathrm{r}}{ }^{\mathrm{c}} \mathrm{G}_{\mathrm{r}}{ }^{\mathrm{s}} \frac{\Omega \delta}{\mathrm{c}}\left(\frac{\pi \delta v}{\omega} \mathrm{A}_{\mathrm{cm}}\right) .
$$

In comparison, the photocurrent from an arm cavity signal is (Appendix B)

$$
\mathrm{I}=\frac{1}{2} \epsilon \frac{\mathrm{e}}{\mathrm{h} v_{0}} \Gamma \mathrm{Pt}_{\mathrm{r}}{ }^{2} \mathrm{G}_{\mathrm{r}}{ }^{\mathrm{c}} \mathrm{G}_{\mathrm{r}} \frac{\Omega \delta}{\mathrm{c}}\left(\mathrm{G}_{\mathrm{a}} \mathrm{kx}_{\mathrm{a}-}\right) .
$$

The signal from shot noise that is due to sideband power at the dark port is

$$
\mathrm{I}_{\mathrm{s}}=\mathrm{e}\left[\frac{4 \epsilon \mathrm{P}}{\mathrm{h} v_{0}}\left(\mathrm{t}_{\mathrm{r}} \mathrm{G}_{\mathrm{r}} \frac{\Omega \delta}{\mathrm{c}} \frac{\Gamma}{2}\right)^{2}\right]^{1 / 2} .
$$

We use the values from Table 4 (Appendix D) for the interferometer parameters and imperfections. In Table 3 we list the noise sources and the imperfections to which they couple to produce gw noise. The third column lists the value of each noise source that, when coupled through the imperfection listed in the second column, produces a gw noise of $10^{-20} \mathrm{~m} / \sqrt{\mathrm{Hz}}$ at $100 \mathrm{~Hz}(10 \%$ of the LIGO design sensitivity).

\section{Discussion of Noise}

As noted above, the filtering of the coupled recycling-arm cavity, a factor of $\sim 100$ at $100 \mathrm{~Hz}$, applies to noise about the carrier but not to the rf sidebands. All the noise couplings listed in Table 3 from the laser and the oscillator are due to noise on the rf sidebands. We also find that oscillator phase noise at the demodulation does not give rise to an output noise, as the time-varying demodulation follows exactly a variation on the phase of the rf sidebands.

Both laser frequency noise (coupling to a loss mismatch of the arm cavities that allows phase-shifted dc carrier light to appear at the dark port and beat against the frequency noise audio sidebands on the rf sidebands) and laser amplitude noise (coupling to a differential arm cavity deviation from exact resonance, which allows phaseshifted dc carrier light to appear at the dark port and beat against the amplitude noise audio sidebands on the rf sidebands) must be suppressed to ensure that the unfil- 
tered noise that appears at the signal output is at the re quired level. The values used in Table 3 for laser frequency and amplitude noise, which require external stabilization, are within the expected LIGO 10-W Iaser performance. The arm cavity reflectivity match of $0.5 \%$ is satisfied with the expected surface uniformity and losses of the cavity optics, ${ }^{10}$ and the requirements of arm cavity and recycling cavity length deviations of $10^{-13}$ and $10^{-11} \mathrm{~m}$ are met with the LIGO length-control design. The oscillator phase noise requirement is satisfied with a currently available commercial unit, ${ }^{11}$ but external amplitude stabilization for the oscillator will be necessary. Finally, the specified level of backscattered light requires the use of beam tube baffles for the arm cavity scattered light and properly chosen photodiode surface quality for dark port scatter.

\section{CONCLUSIONS}

We have presented a method by which the effect of noise on the input light of a gravitational wave interferometer can be rigorously determined. The method involves expressing the noise as audio sidebands about the carrier and rf sideband frequency, determining the frequencydependent transfer function of the interferometer, propagating the light spectrum through the frequency dispersive interferometer cavities to the signal output, and interfering the light at the photodetector and demodulating the signal output. The appearance of noise after the mode cleaner or at the gw output is coupled to scattered light or to frequency and amplitude noise from the laser and the oscillator and to interferometer imperfections such as cavity deviations from resonance or arm cavity loss mismatches. Using LIGO as an example, we have determined the noise couplings and excess noise after the mode cleaner and the gw strain noise. We found that for the LIGO configuration the gw sensitivity is generally limited by the noise on the rf sidebands. Finally, this method may be applied to the noise analysis of alternate gw interferometer configurations. ${ }^{12}$

\section{APPENDIX A: ARM CAVITY CARRIER REFLECTIVITY}

With the circulating field analysis of Fig. 6 we can write

$$
r^{c}=r_{f}+\frac{t_{f}^{2} \exp (-i \Phi)}{1+r_{f} \exp (-i \Phi)}
$$

where

$$
\begin{gathered}
\Phi=2\left(\mathrm{k}+\frac{\omega}{\mathrm{c}}\right)\left(\mathrm{L}+\mathrm{dx}_{\mathrm{a}}\right)=\pi+\frac{2 \mathrm{~L} \omega}{\mathrm{C}}+2 \mathrm{kdx}_{\mathrm{a}}, \\
\left.r^{c}=\left.\frac{E_{r}}{E_{i}} \quad \stackrel{E_{i}}{\underset{E_{r}}{\mathrm{~L}+d x_{a}}}\right|_{r_{f}} ^{\longrightarrow}\right)
\end{gathered}
$$

Fig. 6. Reflectivity of the arm cavity for the carrier field. The input mirror reflectivity is $r_{f}$, and the end mirror reflectivity is 1 .

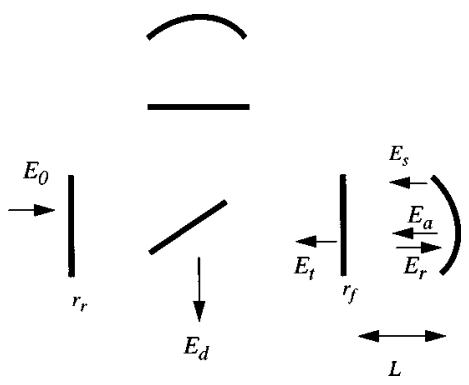

Fig. 7. Arm cavity circulating fields. $E_{s}$ is a source field of audio sidebands from arm cavity motion.

$r^{c}$ is the carrier reflectivity, $L$ is the arm cavity length, $\omega$ is the audio sideband frequency, $r_{f}$ and $t_{f}$ are the arm cavity input mirror reflectivity and transmission, respectively, and $\mathrm{dx}_{\mathrm{a}}$ is the cavity deviation from resonance. We have assumed the end mirror reflectivity to be unity. Thus

$$
r^{c}=\frac{r 0+i\left(r_{f} \frac{\omega}{\omega_{c}}+k G_{a} d x_{a}\right)}{1+i \frac{\omega}{\omega_{c}}},
$$

where $\omega_{c}=(c / 2 L)\left(1-r_{f}\right), \quad G_{a}=2 /\left(1-r_{f}\right)$, and $r 0$ $=r^{C}(\Phi=\pi)$ is the arm cavity reflectivity on exact resonance. Note that with input mirror loss $\mathrm{I}_{\mathrm{f}} \ll \mathrm{t}_{\mathrm{f}}$, where $\mathrm{I}_{\mathrm{f}}^{2}+\mathrm{r}_{\mathrm{f}}^{2}+\mathrm{t}_{\mathrm{f}}^{2}=1$, we have $\mathrm{r} 0 \sim-1$.

\section{APPENDIX B: ARM CAVITY SIGNAL}

We solve for the circulating field in the recycled intensive field operation, where the audio sidebands $E_{s}$ generated from the arm cavity motion $x_{a} \cos (\omega t)$ are seen as a source field to be added to the circulating field $\mathrm{E}_{\mathrm{a}}$ (see Fig. 7):

$$
E_{a}=E_{s}+E_{r}=E_{s}+E_{a} \exp \left[-i 2\left(k+\frac{\omega}{c}\right) L\right]\left(-r_{f}\right)
$$

so that

$$
E_{a}=\frac{E_{s}}{\left(1-r_{f}\right)\left(1+\frac{i \omega}{\omega_{c}}\right)}
$$

and

$$
E_{d}=\frac{E_{s} t_{f}}{\left(1-r_{f}\right)\left(1+\frac{i \omega}{\omega_{c}}\right)}
$$

Now $E_{s}=$ source field of audio sidebands:

$$
=\frac{E_{0}}{2} t_{r} G_{r} c\left(\frac{t_{f}}{1-r_{f}}\right)\left\{1+i k x_{a}[\exp (i \omega t)+\exp (-i \omega t)]\right\} \text {. }
$$

Thus

$$
E_{d}=\frac{E_{0}}{2} t_{r} G_{r}{ }^{c} G_{a} \frac{\left\{1+i k x_{a}[\exp (i \omega t)+\exp (-i \omega t)]\right\}}{\left(1+\frac{i \omega}{\omega_{c}}\right)} \text {. }
$$




\section{APPENDIX C: DEMODULATION OF THE PHOTOCURRENT}

1. Photodetector Current

If $E_{1}\left(\omega_{1}+\omega_{0}\right)$ and $E_{2}\left(\omega_{2}+\omega_{0}\right)$ are two electric fields incident on the photodetector, where $\omega_{1}>\omega_{2}$ and $\omega_{1}, \omega_{2}$ $\ll \omega_{0}$ is the optical frequency, we can write

$$
\begin{aligned}
\mathrm{E} & =\operatorname{Re}\left\{\left[\mathrm{E}_{1} \exp \left(\mathrm{i} \omega_{1} \mathrm{t}\right)+\mathrm{E}_{2} \exp \left(\mathrm{i} \omega_{2} \mathrm{t}\right)\right] \exp \left(\mathrm{i} \omega_{0} \mathrm{t}\right)\right\}, \\
\mathrm{i}_{\mathrm{p}} & =\overline{\mathrm{E} \mathrm{E}^{*}}=\left\{\mathrm{E}_{1} \mathrm{E}_{2}^{*} \exp \left[\mathrm{i}\left(\omega_{1}-\omega_{2}\right) \mathrm{t}\right]+\mathrm{c} \cdot \mathrm{c} .\right\} \\
& =2 \operatorname{Re}\left\{\mathrm{E}_{1} \mathrm{E}_{2}^{*} \exp \left[\mathrm{i}\left(\omega_{1}-\omega_{2}\right) \mathrm{t}\right]\right\},
\end{aligned}
$$

where $i_{p}$ is the photodetector current and we have omitted terms that do not mix the two frequencies.

Referring to Fig. 2 and writing the contributions to $i_{p}$ for all the fields separated by $\Omega \pm \omega$ that will give a demodulated in-band output, we have

$$
\begin{aligned}
\mathrm{i}_{\mathrm{p}}= & 2 \operatorname{Re}\left\{\mathrm{E}_{00} \mathrm{E}_{-1-1}^{*} \exp [\mathrm{i}(\Omega+\omega) \mathrm{t}]\right. \\
& +\mathrm{E}_{00} \mathrm{E}_{-11}^{*} \exp [\mathrm{i}(\Omega-\omega) \mathrm{t}] \\
& +\mathrm{E}_{11} \mathrm{E}_{00}^{*} \exp [\mathrm{i}(\Omega+\omega) \mathrm{t}] \\
& +\mathrm{E}_{1-1} \mathrm{E}_{00}^{*} \exp [\mathrm{i}(\Omega-\omega) \mathrm{t}] \\
& +\mathrm{E}_{0-1} \mathrm{E}_{-10}^{*} \exp [\mathrm{i}(\Omega-\omega) \mathrm{t}] \\
& +\mathrm{E}_{10} \mathrm{E}_{0-1}^{*} \exp [\mathrm{i}(\Omega+\omega) \mathrm{t}] \\
& +\mathrm{E}_{01} \mathrm{E}_{-10}^{*} \exp [\mathrm{i}(\Omega+\omega) \mathrm{t}] \\
& \left.+\mathrm{E}_{10} \mathrm{E}_{01}^{*} \exp [\mathrm{i}(\Omega-\omega) \mathrm{t}]\right\},
\end{aligned}
$$

where the first four terms represent mixing of the carrier with the rf audio sidebands and the second four terms represent mixing of the carrier audio sidebands with the rf sidebands.

\section{Demodulation}

We consider demodulation both in phase with the modulation of Eq. (4) and out of phase (also referred to as quadrature phase). The mixer output $\mathrm{V}$ is

Quadrature phase:

$$
\begin{aligned}
V= & \int i_{p} \sin \Omega t d t \\
V= & -\operatorname{Im}\left[E_{00}\left(E_{-1-1}^{*}-E_{1-1}^{*}\right)+E_{00}^{*}\left(E_{11}-E_{-11}\right)\right. \\
& +E_{01}\left(E_{-10}^{*}-E_{10}^{*}\right) \\
& \left.+E_{0-1}^{*}\left(E_{10}-E_{-10}\right) \exp (i \omega t)\right]
\end{aligned}
$$

and therefore

$$
\begin{aligned}
\operatorname{Mag}[\mathrm{V}]=\mid \mathrm{E}_{00}\left(\mathrm{E}_{-1-1}^{*}-\mathrm{E}_{1-1}^{*}\right)+\mathrm{E}_{00}^{*}\left(\mathrm{E}_{11}-\mathrm{E}_{-11}\right) \\
+\mathrm{E}_{01}\left(\mathrm{E}_{-10}^{*}-\mathrm{E}_{10}^{*}\right)+\mathrm{E}_{0-1}^{*}\left(\mathrm{E}_{10}-\mathrm{E}_{-10}\right) \mid
\end{aligned}
$$

In phase:

$$
V=\int i_{p} \cos \Omega t d t
$$

\begin{tabular}{|c|c|c|}
\hline Symbol & Description & Nominal value \\
\hline $\mathrm{P}$ & Laser power & $6 \mathrm{~W}$ \\
\hline$v_{0}$ & Laser frequency & $3 \times 10^{14} \mathrm{~Hz}$ \\
\hline $\mathrm{E}_{0}$ & Laser electric field & \\
\hline$\omega$ & Noise audio sideband frequency & $100 \mathrm{~Hz}$ \\
\hline$\Gamma$ & rf oscillator modulation depth & $0.5 \mathrm{rad}$ \\
\hline$\Omega$ & rf oscillator angular frequency & $(2 \pi) 25 \mathrm{MHz}$ \\
\hline k & Laser wave number & $(2 \pi) 10^{6} \mathrm{~m}^{-1}$ \\
\hline$x_{s}$ & Seismic motion at $100 \mathrm{~Hz}$ & $10^{-11} \mathrm{~m} / \sqrt{\mathrm{Hz}}$ \\
\hline $\mathrm{E}_{\text {scat }}$ & Scattered light electric field & \\
\hline $\mathrm{E}_{\mathrm{m}}$ & Mode cleaner output electric field & \\
\hline$E_{d}$ & Dark port electric field & \\
\hline$r_{m}$ & Mode-cleaner mirror reflectivity & 0.999 \\
\hline $\mathrm{L}_{\mathrm{m}}$ & Mode-cleaner length & $12 \mathrm{~m}$ \\
\hline$d x_{m}$ & Mode-cleaner rms length offset & $10^{-13} \mathrm{~m}$ \\
\hline $\mathrm{d} \Omega$ & $\begin{array}{l}\text { Sideband detuning from mode-cleaner } \\
\text { resonance }\end{array}$ & $(2 \pi) 100 \mathrm{~Hz}$ \\
\hline$\omega_{\mathrm{c}}$ & Mode-cleaner pole frequency & $4 \mathrm{kHz}$ \\
\hline$r_{r}$ & Recycling-mirror reflectivity & 0.985 \\
\hline$r_{f}$ & Arm cavity input mirror reflectivity & 0.985 \\
\hline$r^{c}$ & Arm cavity carrier reflectivity & \\
\hline$\omega_{\mathrm{c}}$ & Arm cavity pole frequency & $(2 \pi) 90 \mathrm{~Hz}$ \\
\hline$\omega_{\mathrm{cc}}$ & Coupled cavity pole frequency & $(2 \pi) 1 \mathrm{~Hz}$ \\
\hline ro & $\begin{array}{l}\text { Arm cavity carrier reflectivity at exact } \\
\text { resonance }\end{array}$ & -0.99 \\
\hline \multirow[t]{2}{*}{$\mathrm{L}$} & Arm cavity length & $4000 \mathrm{~m}$ \\
\hline & Total arm cavity loss & 150 ppm \\
\hline$d x_{a-}$ & Arm cavity rms differential offset & $10^{-13} \mathrm{~m}$ \\
\hline $\mathrm{dx}_{\mathrm{r}-}$ & Recycling cavity rms differential offset & $10^{-11} \mathrm{~m}$ \\
\hline$\delta$ & $\begin{array}{l}\text { Asymmetry (difference in recycling } \\
\text { cavity lengths) }\end{array}$ & $0.4 \mathrm{~m}$ \\
\hline$A_{c m}$ & Arm cavity carrier reflectivity match & 0.005 \\
\hline$\epsilon$ & Photodector quantum efficiency & 0.85 \\
\hline
\end{tabular}

$$
\begin{aligned}
\operatorname{Mag}[V]=\mid E_{00}\left(E_{-1-1}^{*}+E_{1-1}^{*}\right)+E_{00}^{*}\left(E_{11}+E_{-11}\right) \\
\\
+E_{01}\left(E_{-10}^{*}+E_{10}^{*}\right)+E_{0-1}^{*}\left(E_{10}+E_{-10}\right) \mid .
\end{aligned}
$$

3. Demodulation Phase Noise Phase noise on the local oscillator demodulation produces audio sidebands about the demodulation frequency.

The quadrature demodulation spectrum is

$$
\mathrm{A}=\Gamma\left\{\sin \Omega \mathrm{t}+\left(\mathrm{a}_{0} / 2\right)[\cos (\Omega+\omega) \mathrm{t}+\cos (\Omega-\omega) \mathrm{t}]\right\} .
$$

Then the mixer output is

$$
\begin{aligned}
V= & \int \operatorname{Re}\left[\left(\mathrm{E}_{00} \mathrm{E}_{-10}^{*}+\mathrm{E}_{10} \mathrm{E}_{00}^{*}\right) \exp (\mathrm{i} \Omega \mathrm{t})\right] \frac{\mathrm{a}_{0}}{2}[\cos (\Omega \\
& +\omega) \mathrm{t}+\cos (\Omega-\omega) \mathrm{t}] \mathrm{dt} \\
= & -\operatorname{Re}\left(\mathrm{E}_{00} \mathrm{E}_{-10}^{*}+\mathrm{E}_{10} \mathrm{E}_{00}^{*}\right) \mathrm{a}_{0} \cos (\omega \mathrm{t}) .
\end{aligned}
$$

\section{APPENDIX D: LIST OF SYMBOLS AND NOMINAL VALUES}

In Table 4 we list the symbols used in this paper and their nominal values in the initial LIGO configuration.

\section{Table 4. Symbols and Their Nominal Values in the Initial LIGO Configuration}




\section{ACKNOWLEDGMENTS}

The authors acknowledge the seminal work of Rainer Weiss and Martin Regehr in analyzing the noise performance of the interferometer configuration studied in this research. Albert Lazzarini provided useful comments regarding the manuscript. This research was supported by the National Science Foundation under cooperative agreement PHY-9210038.

J . B. Camp's e-mail address is jordan@igo.caltech.edu.

\section{REFERENCES AND NOTES}

1. A. Abramovici, W. Althouse, R. W. P. Drever, Y. Gursel, S. Kawamura, F. J. Raab, D. Shoemaker, L. Sievers, R. E. Spero, R. E. Vogt, R. Weiss, S. E. Whitcomb, and M. E. Zucker, "LIGO: The Laser Interferometer GravitationalWave Observatory," Science 256, 325-333 (1992).

2. A. Giazotto, "The VIRGO experiment: status of the art," in First Edoardo Amaldi Conference on Gravitational Wave Experiments, E. Coccia, G. Pizella, and F. Ronga, eds. (World Scientific, Singapore, 1995), pp. 86-99.

3. K. Tsubono, "300-m laser interferometer gravitational wave detector (TAMA300) in J apan," in First Edoardo Amaldi Conference on Gravitational Wave Experiments, E. Coccia, G. Pizella, and F. Ronga, eds. (World Scientific, Singapore, 1995), pp. 112-114.
4. K. Danzmann, "GEO600-a 600 m laser interferometric gravitational wave antenna," in First Edoardo Amaldi Conference on Gravitational Wave Experiments, E. Coccia, G. Pizella, and F. Ronga, eds. (World Scientific, Singapore, 1995), pp. 100-111.

5. R. W. P. Drever, J. L. Hall, F. V. Kowalski, J. Hough, G. M. Ford, A. J. Munley, and H. Ward, "Laser phase and fre quency stabilization using an optical resonator," Appl. Phys. B 31, 97-105 (1983).

6. R. Drever, in Quantum Optics, Experimental Gravity and Measurement Theory, P. Meystre and M. Scully, eds. (Plenum, New York, 1983), pp. 503-514.

7. A. Siegman, Lasers (University Science, Mill Valley, Calif., 1986), Chap. 11.

8. M. Regehr, "Signal extraction and control for an interferometric gravitational wave detector," Ph.D. dissertation (California Institute of Technology, Pasadena, Calif., 1995).

9. K. D. Skeldon and K. A. Strain, "Response of a Fabry-Perot optical cavity to phase-modulation sidebands for use in electro-optic control systems," Appl. Opt. 36, 6802-6808 (1997).

10. S. Whitcomb, "Optics Development for LIGO," in Proceedings of the TAMA International Workshop on Gravitational Wave Detection, K. Tsubono, M.-K. Fujimoto, and K. Kuroda, eds. (Universal Academy Press, Tokyo, 1996), pp. 229-239.

11. Marconi model 2023.

12. D. E. McClelland, J . B. Camp, J . Mason, W. Kells, and S. E. Whitcomb, "Arm cavity resonant sideband control for laser interferometric gravitational wave detectors," Opt. Lett. 24, 1014-1016 (1999). 